\title{
LETTER
}

\section{High-sensitivity infrared vibrational nanospectroscopy in water}

\author{
Mingzhou Jin, Feng Lu and Mikhail A Belkin \\ Light: Science \& Applications (2017) 6, e17096; doi:10.1038/lsa.2017.96; published online 28 July 2017
}

Mid-infrared vibrational spectroscopy is a universal label-free tool for identifying molecular compounds in chemical and biological samples on the basis of their 'fingerprint' vibrational absorption lines. Vibrational spectroscopy with nanometer spatial resolution can reveal the chemical composition of samples at the nanoscale, and several scanning-probe techniques have been developed to address this need ${ }^{1-22}$. It is also highly desirable to study biological and chemical samples in their native aqueous environments rather than in air. In aqueous environments, however, the sensitivity of the current vibrational nanospectroscopy techniques deteriorates dramatically ${ }^{7,23}$. Here, we report the first mid-infrared nanospectroscopy technique that retains nanoscale sensitivity and spatial resolution when the sample and the scanning probe are completely immersed in water. This method overcomes challenges including water absorption and scattering and the mechanical damping of cantilever vibrations. We further demonstrate spectroscopy and imaging of 20- to $50-\mathrm{nm}$ thick polymer samples with a $25-\mathrm{nm}$ spatial resolution in the biologically relevant Amide I and II spectral regions.

The diffraction limit restricts the spatial resolution of far-field midinfrared $(\lambda \approx 3-15 \mu \mathrm{m})$ microscopy to the micrometer scale. To perform mid-infrared spectroscopy at the nanoscale, three major techniques have been developed: near-field scanning optical microscopy $\left(\mathrm{NSOM}^{1-9,21}\right)$, infrared photoexpansion nanospectroscopy $\left(\text { AFM-IR }{ }^{10-18,22} \text { ) and photoinduced force microscopy (PiFM) }\right)^{19,20}$. For operation in air, all these techniques have demonstrated a sensitivity of or close to a molecular monolayer and a spatial resolution of $10-30 \mathrm{~nm}$, which is principally limited by the apex radius of the atomic force microscope (AFM) tip.

However, the performance changes when a sample is immersed in water. The scattering and absorption of infrared light and the mechanical damping of cantilever oscillations by water lead to a severe degradation of the performance of all current vibrational nanospectroscopy techniques. Current state-of-the-art results include imaging of a 6 - $\mu \mathrm{m}$-diameter Melamine beads in water with $\sim 1 \mu \mathrm{m}$ spatial resolution using aperture-type $\mathrm{NSOM}^{7}$ and a photoexpansion nanospectroscopy of $\sim 1-\mu \mathrm{m}$-thick Candida albicans fungi ${ }^{23}$ in $850-1250 \mathrm{~cm}^{-1}$ spectral range. Scattering-type NSOM imaging of samples in ultrathin graphene-covered liquid cells has also recently been demonstrated ${ }^{3}$. In this case, the sample was covered by an $\sim 10$ $\mathrm{nm}$ layer of water below a graphene sheet, and the AFM probe was operated in air. However, ultrathin graphene liquid cells are difficult to implement and are known to subject samples to very high-hydrostatic pressure (ca. $1 \mathrm{GPa})^{24}$.

Here, we demonstrate that high-sensitivity infrared nanospectroscopy can be performed in bulk liquid cells, thus avoiding the complications associated with nanoscale liquid cells. Our method is based on the resonantly enhanced infrared photoexpansion nanospectroscopy (REINS) technique, which we have recently developed ${ }^{18}$. The schematic of our experimental setup is shown in Figure 1a. We illuminate the sample with a train of optical pulses from a quantum cascade laser. The sample is positioned on top of the prism for evanescent illumination, and a gold-coated silicon tip is placed in contact with the sample. A cover glass slide, attached at the back of the cantilever chip holder, holds a water droplet between the glass slide and prism.

REINS operates by detecting the cantilever oscillation amplitude produced by the photoexpansion of a sample. The sample absorbs laser light and undergoes rapid thermal expansion. The expansion produces a force on the AFM tip that leads to a small cantilever deflection detectable by the AFM position-sensitive photodetector (PSPD); see Figure 1a. The laser pulses are sent in resonance with the cantilever oscillation, and the induced cantilever oscillation amplitude $\Delta z$ is given by:

$$
\Delta z \propto \alpha_{\mathrm{abs}} \cdot \eta \cdot Q \cdot I
$$

where $\alpha_{\mathrm{abs}}$ is the sample absorption coefficient, $\eta$ is the optical field enhancement in the sample below the tip, Q is the quality factor (Q-factor) of the cantilever oscillation, and $I$ is the input light intensity. Assuming that the field enhancement is constant across the spectral range of interest, the cantilever oscillation amplitude, normalized by the laser intensity, is directly proportional to the sample absorption $\alpha_{\mathrm{abs}}{ }^{18}$.

In an aqueous environment, mid-infrared nanospectroscopy techniques encounter two major challenges. One is the strong midinfrared light scattering and absorption in water, which can overwhelm the signal from the sample. REINS detects sample absorption via a non-optical method, and unlike NSOM, it is insensitive to light 
a

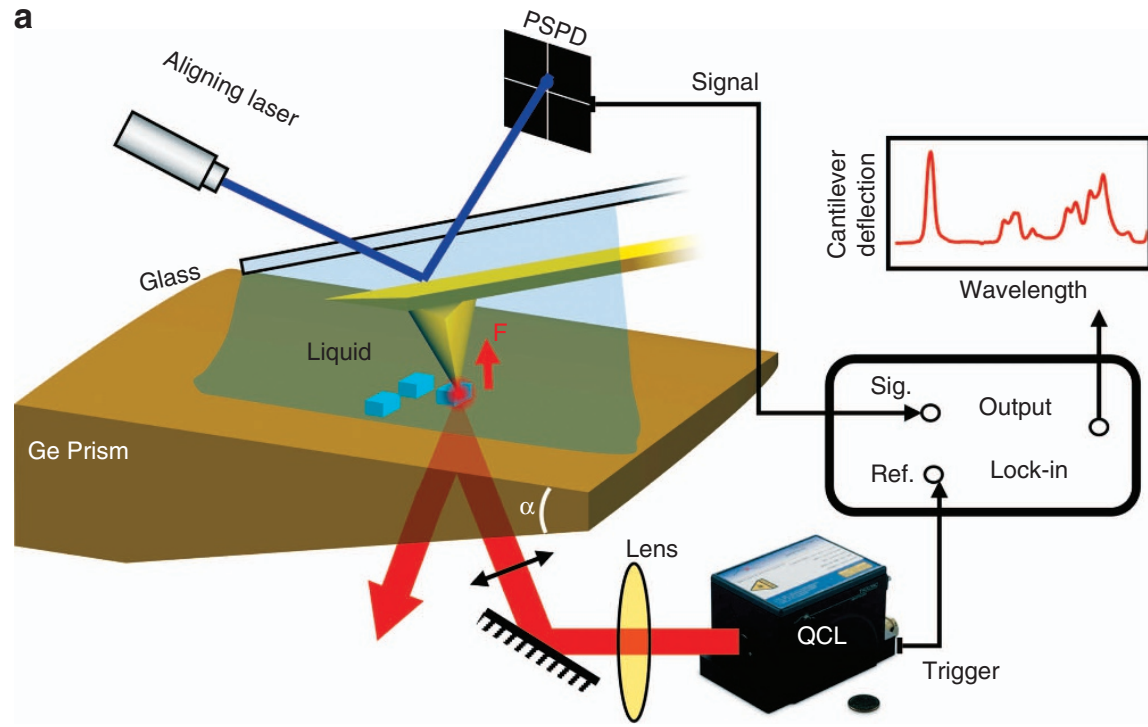

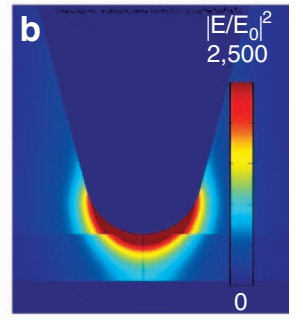

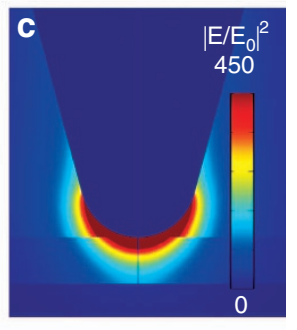

Figure 1 Experimental setup and tip-enhancement simulations. (a) Experimental setup for the photoexpansion nanospectroscopy in liquid. The polarization of the incident light is parallel to the incident plane. The wedge angle $\alpha$ is $14.5^{\circ}$. (b, c) Optical field intensity enhancement below the metallized AFM in the case of a sample (PMMA) on top of a germanium prism (b) and a ZnSe prism (c).

scattering ${ }^{17,18}$. We suppress the water absorption by nanofocusing the laser light onto the sample volume immediately below the AFM tip. To achieve this, the sample is evanescently illuminated using a p-polarized laser light, and the optical field intensity in the sample below the AFM tip is enhanced because of a combination of the electrostatic boundary condition effect at the prism-sample interface ${ }^{25}$ and the tip enhancement due to the metallized AFM tip ${ }^{18,26}$, as shown in Figure $1 \mathrm{~b}$. We use a prism made of germanium, which has the highest dielectric constant $\left(\varepsilon_{r}^{G e} \approx 16\right)$ of all the mid-infrared materials and provides the strongest field enhancement. The importance of a germanium prism is illustrated in Figure 1c, which shows the optical field intensity below the AFM tip for the case of a ZnSe prism $\left(\varepsilon_{r}^{Z n S e} \approx 5.8\right.$ ), which has a significantly lower dielectric constant than germanium and provides $\sim 6$ times lower intensity enhancement. This point is further explained in Supplementary Information.

The second challenge faced by the mid-infrared nanospectroscopy techniques in water is strong mechanical damping of the cantilever oscillations. The Q-factor of the fundamental cantilever mechanical resonance can decrease from $\sim 100$ in air to only $\sim 1$ in water ${ }^{27}$. The resonance peak broadening makes it very challenging to operate an AFM in non-contact or tapping modes ${ }^{28}$. In contrast to the NSOM and photoinduced force microscopy techniques, REINS records sample spectra by operating the AFM in contact mode ${ }^{18}$, thereby avoiding this problem. Liquid damping does affect REINS through the decrease in the Q-factor; see Equation (1). To boost the signal level, we tune the rate of laser pulses to excite the higher-order cantilever flexural modes that are expected to have higher Q-factors in liquid.

The experimentally measured cantilever mechanical resonances in water and their Q-factors are shown in Figure 2. For the cantilever flexural modes above the 4 th mode, the cantilever slope varies significantly over the $40-\mu \mathrm{m}$ aligning laser spot diameter, thus resulting in a decrease in the PSPD signal, which limited our setup to the 4th flexural mode with a Q-factor of 9.9.

Figure $1 \mathrm{~b}$ shows that despite the nanofocusing, a small fraction of the liquid surrounding the tip may still be exposed to a high-intensity optical field, thus resulting in significant liquid photoexpansion. Heavy water $\left(\mathrm{D}_{2} \mathrm{O}\right)$, compared with water $\left(\mathrm{H}_{2} \mathrm{O}\right)$, has a much smaller

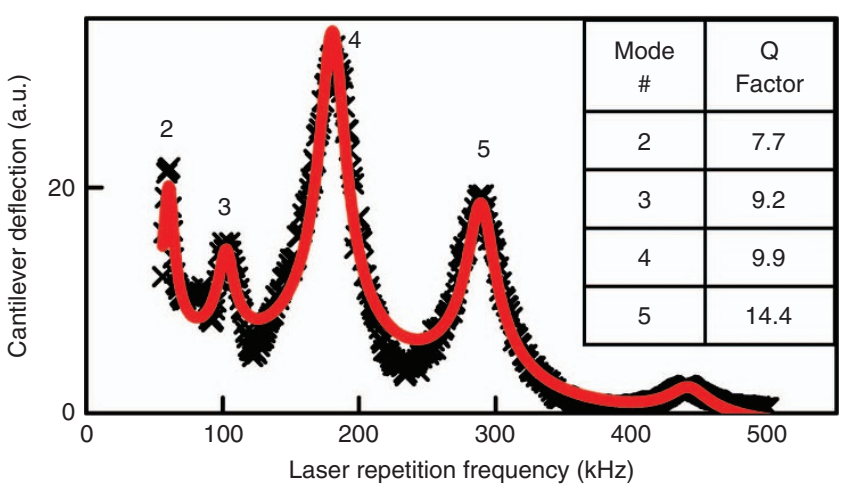

Figure 2 Experimentally measured cantilever vibrational spectrum in $D_{2} O$ (black crosses), fitted using five resonant modes (red curve), as discussed in Supplementary Information. The Q-factors of the four modes are listed in the inset.

absorption coefficient in the Amide I and II bands ${ }^{29}$ (see Supplementary Information). Therefore, we chose to operate in the $\mathrm{D}_{2} \mathrm{O}$ environment.

For a proof-of-concept demonstration, we fabricated nanoscale patterns of 20- and 50-nm-thick layers of Poly(methyl methacrylate) (PMMA) on top of the Ge prism. The spectra were obtained by normalizing the cantilever oscillation amplitude to the laser intensity (cf. Equation (1)) and subtracting the $\mathrm{D}_{2} \mathrm{O}$ background signal, as discussed in the Supplementary Information. The spectrum of a 20-nm PMMA in $\mathrm{D}_{2} \mathrm{O}$ is plotted in Figure 3a. It features a sharp peak at $1730 \mathrm{~cm}^{-1}$, corresponding to the $\mathrm{C}=\mathrm{O}$ stretching mode of PMMA. The signal-to-noise ratio of these measurements was estimated to be $\sim 5$. Figure $3 \mathrm{a}$ also shows an infrared absorption spectrum of bulk PMMA, which is in excellent agreement with the measured spectrum.

To demonstrate the spatial resolution of our technique, we performed spectroscopy and imaging of a 50-nm-thick PMMA pattern on a germanium prism. Figure $3 \mathrm{~b}$ exhibits the AFM topography of a single 'UT' logo. We took the spectra at 30 points, at $25-\mathrm{nm}$ intervals, along the arrow in Figure $3 \mathrm{~b}$. The selected spectra are shown in 
a

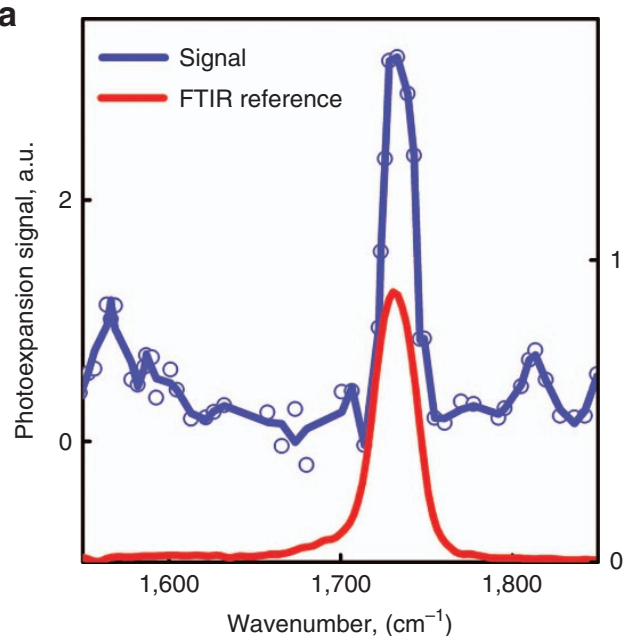

d

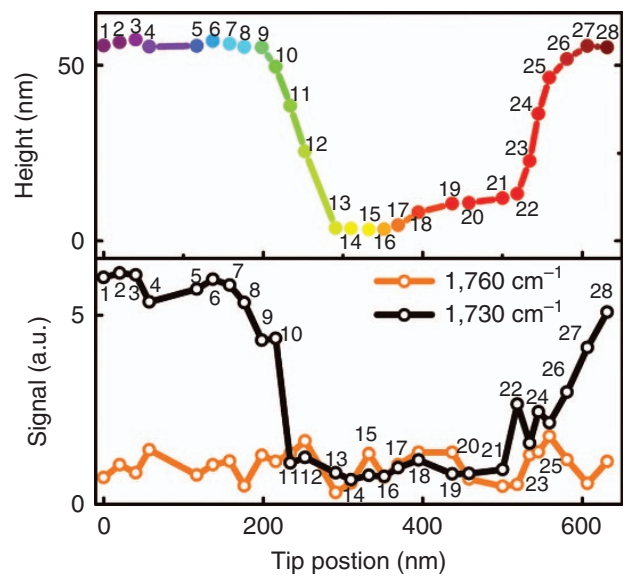

b

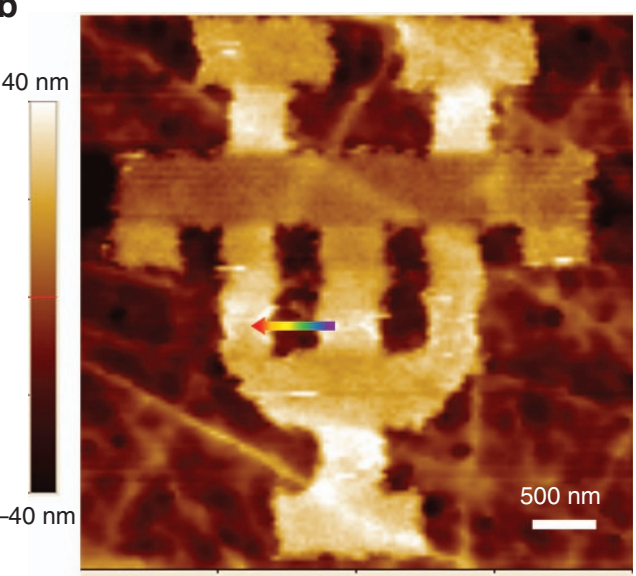

e

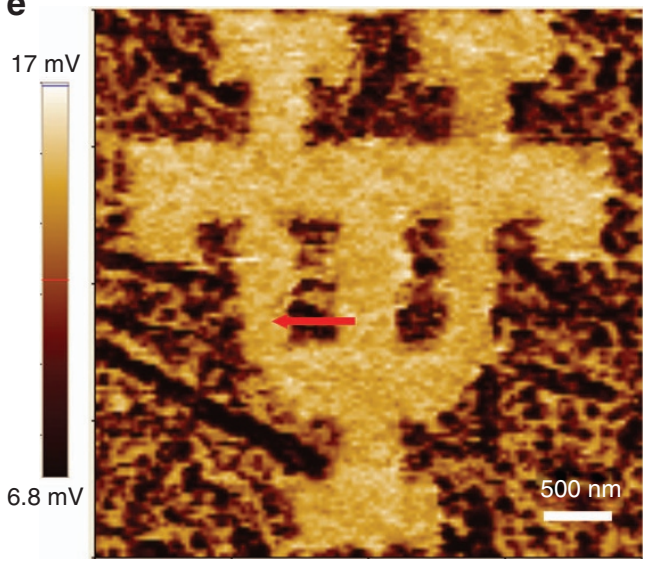

C

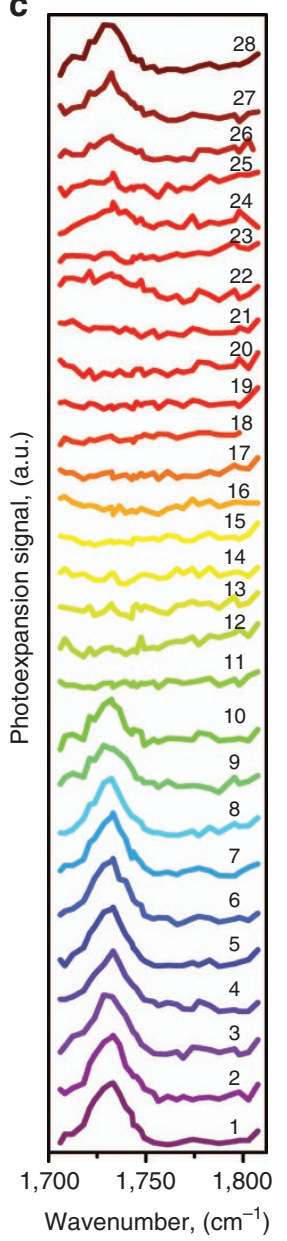

Figure 3 REINS spectra and chemical nano-imaging. (a) Normalized REINS spectrum of a 20-nm-thick PMMA film in $\mathrm{D}_{2} \mathrm{O}$ vs the reference Fourier transform infrared spectroscopy (FTIR) absorption spectrum of PMMA. (b) AFM topography of a 50-nm-thick PMMA pattern. Bright areas are the PMMA film, and dark areas are the bare germanium. (c) REINS spectra, taken at 25-nm intervals along the arrow shown in b. (d) Topography (top) and the REINS signal (bottom) at $1730 \mathrm{~cm}^{-1}$ (black) and $1760 \mathrm{~cm}^{-1}$ (orange), taken at 25-nm intervals along the scan line shown in $\mathbf{b}$. (e) Chemical mapping of the sample area shown in $\mathbf{b}$.

Figure 3c. The PMMA absorption peak appeared only when the tip was on top of the PMMA. To further demonstrate the nanoscale resolution of our technique, Figure $3 \mathrm{~d}$ plots the photoexpansion signal strength at the laser wavenumber of $1730 \mathrm{~cm}^{-1}$ (at the PMMA peak) and at $1760 \mathrm{~cm}^{-1}$ (away from the PMMA peak) against the sample topography as a function of the tip position. The contrast was observed only at the PMMA absorption peak at $1730 \mathrm{~cm}^{-1}$, and the signal followed the shape of the topographical line scan.

Figure $3 e$ shows the mid-infrared chemical mapping. In this case, we set our laser wavelength to the PMMA infrared absorption peak and measured the cantilever deflection signal as a function of the tip position. The area imaged in Figure $3 \mathrm{e}$ was the same as that of the topographic image in Figure $3 \mathrm{~b}$, and a clear contrast in the PMMA patterns was observed.

Figure $4 \mathrm{a}$ shows the simulated temperature distribution in a $20-\mathrm{nm}$ PMMA sample and in the surrounding $\mathrm{D}_{2} \mathrm{O}$ at the end of a laser pulse. Our simulations showed that the sample experiences a maximum temperature increase of $\Delta T=2.8 \mathrm{~K}$ at the end of the laser pulse, which is comparable to the $\Delta T=5.9 \mathrm{~K}$ calculated for the monolayer sample on gold-coated substrates by using the REINS technique in air $^{18}$. Figure $4 \mathrm{~b}$ shows that $\mathrm{D}_{2} \mathrm{O}$ heating was present, owing to liquid absorption, even when the sample was non-absorbing. The heating

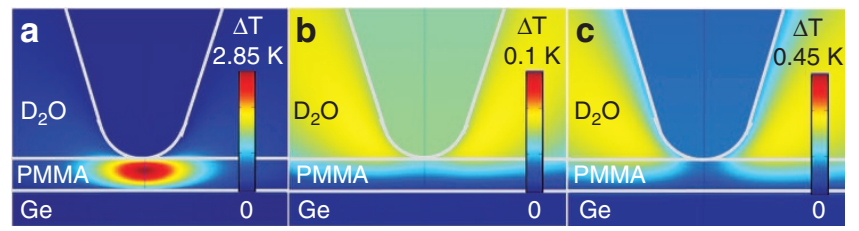

Figure 4 Temperature increase in the sample during the laser pulse. Simulated temperature distribution in the 20-nm-thick PMMA sample at the end of a 300-ns quantum cascade laser pulse with a pulse intensity of approximately $3.9 \mathrm{~kW} \mathrm{~cm}^{-2}$, assuming (a) a PMMA absorption coefficient of $\alpha_{a b s}=5400 \mathrm{~cm}^{-1}$ (corresponding to the peak PMMA absorption at $1730 \mathrm{~cm}^{-1}$ ) at the laser wavelength and the sample being immersed in $\mathrm{D}_{2} \mathrm{O}$, (b) PMMA not absorbing $\left(\alpha_{\mathrm{abs}}=0\right)$ at the laser wavelength and the sample being immersed in $\mathrm{D}_{2} \mathrm{O}$, and (c) PMMA not absorbing $\left(\alpha_{\mathrm{abs}}=0\right)$ at the laser wavelength and the sample being immersed in $\mathrm{H}_{2} \mathrm{O}$.

was significantly higher in the $\mathrm{H}_{2} \mathrm{O}$ environment, as plotted in Figure $4 \mathrm{c}$, thus preventing us from measuring high-quality spectra from the PMMA samples in $\mathrm{H}_{2} \mathrm{O}$ below $100 \mathrm{~nm}$ in thickness (see Supplementary Information).

Despite a similar sample temperature change during the laser pulse, the sensitivity of REINS in $\mathrm{D}_{2} \mathrm{O}$ reported here was a factor of $\sim 10$ less 
than the monolayer sensitivity achieved in $\operatorname{air}^{18}$. The main reason for the difference was a 10-fold decrease in the cantilever Q-factor. This constraint may be overcome in the future by using the encased cantilevers that have recently been reported ${ }^{30}$, in which a hydrophobic encasement maintains an air bubble around the cantilever body. In this way, the water dynamic damping is suppressed, and the high Q-factor is maintained in water.

In conclusion, we have demonstrated a technique for the highsensitivity vibrational nanospectroscopy of chemical samples in an aqueous environment. We achieved a spatial resolution of $25 \mathrm{~nm}$ and obtained spectra of polymer samples as thin as $20 \mathrm{~nm}$. Our work lays a foundation for the infrared nanospectroscopy of chemical and biological samples in their native liquid environments and offers the possibility of in vivo studies of biological samples using infrared nanospectroscopy.

\section{METHODS}

\section{Experimental setup}

A broadly tunable quantum cascade laser (Daylight solutions) with a tuning range of $1470-1970 \mathrm{~cm}^{-1}$ was used as the light source in the setup. We kept the laser duty cycle at $6 \%$ in pulsed mode. The pulse peak power ranged from $80 \mathrm{~mW}$ to $460 \mathrm{~mW}$ depending on the operation wavelength. The laser beam was directed toward the sample by an adjustable gold mirror. During the measurement, the laser operated in a 'sweep scan' mode, in which the laser wavelength was swept continuously, and the laser sent trigger signals at equally spaced wavenumbers. By connecting the wavenumbers to the trigger points, we were able to assign the cantilever deflection signal from the lock-in amplifier to a laser wavenumber. The sweeping speed was adjustable from 0 to $100 \mathrm{~cm}^{-1} \mathrm{~s}^{-1}$. In the experiment, we scanned the laser wavelength at $30 \mathrm{~cm}^{-1} \mathrm{~s}^{-1}$, and the trigger points were set every $1.2 \mathrm{~cm}^{-1}$, with the lock-in integration time set at $30 \mathrm{~ms}$.

The gold-coated silicon AFM probes (ContGB-G from BudgetSensors) had a typical tip radius of $25 \mathrm{~nm}$. Heavy water $(99.99 \%$ Deuterium oxide) was purchased from Sigma-Aldrich. After aligning the optics in air, we used a pipette to deposit a $10-15-\mu 1 \mathrm{D}_{2} \mathrm{O}$ droplet on the target PMMA patterns. Then, we gently moved the AFM probe with the cover slide on top toward the droplet. After the cantilever was completely immersed in the droplet, we readjusted the AFM aligning laser to ensure that the laser spot hit at the end of the cantilever. The PSPD position was also calibrated so that the reflected laser spot is centered on the PSPD.

Polymer samples were positioned on top of optical grade Ge wedge prisms with a top surface roughness of $2 \mathrm{~nm}$. A prism angle of $\alpha=14.5^{\circ}$ (Figure 1a) was chosen so that the incident laser light normal to the cut surface had the critical angle of incidence at the Ge/air interface on top of the prism. In the case of the Ge/polymer and Ge/ water interfaces, the critical angle on the prism surface was in the range of $19-22^{\circ}$, assuming typical values of the refractive index of polymer samples and water in the range of 1.3-1.5. The data presented in the manuscript were collected with a beam incidence angle of $20^{\circ}$ with respect to the surface normal at the prism/sample interface, which corresponds to the incidence angle of $23.5^{\circ}$ with respect to the cut surface of the prism.

\section{Sample preparation}

PMMA patterns on top of the prisms were fabricated via electronbeam lithography. The prism was cleaned via oxygen plasma reactive ion etching and then rinsed with copious amounts of acetone, isopropyl alcohol and deionized water (DI water) to thoroughly remove contaminants. PMMA photoresists of $20 \mathrm{~nm}$ and $50 \mathrm{~nm}$ in thickness were then spin-coated on top of the prism, followed by a 1min prebake at $90{ }^{\circ} \mathrm{C}$. A layer of conductive polymer Espacer $(300 \mathrm{Z}$ Showa Denko) was also spin-coated before electron beam exposure to avoid the charging effect. After electron-beam lithography, the PMMA pattern was developed in a 1:3 MIBK/isopropyl alcohol solution and dried under a nitrogen flow.

\section{Simulations}

Electromagnetic field and temperature increase simulations were performed in 3D using COMSOL Multiphysics. The refractive indices of $\mathrm{D}_{2} \mathrm{O}$ and $\mathrm{H}_{2} \mathrm{O}$ were obtained from Ref. 29 as $1.33+0.005 \mathrm{i}$ and 1.31 $+0.025 \mathrm{i}$ at $1730 \mathrm{~cm}^{-1}$, respectively.

For thermal simulations, we used thermal conductivity coefficients of $60,0.2,0.6$ and $314 \mathrm{~W} \cdot(\mathrm{m} \mathrm{K})^{-1}$ and heat capacities of 1460, 310, 4186 and $129 \mathrm{~J}(\mathrm{~kg} \mathrm{~K})^{-1}$ for the germanium, PMMA, $\mathrm{D}_{2} \mathrm{O}\left(\mathrm{H}_{2} \mathrm{O}\right)$ and gold, respectively.

\section{CONFLICT OF INTEREST}

FL and MAB. are co-authors of US patent 8,869,602, 'High frequency deflection measurement of IR absorption'. MJ declares no competing financial interests.

\section{ACKNOWLEDGEMENTS}

We acknowledge financial support from the Robert A. Welch Foundation (grant no. F-1705) and the US Department of Energy STTR program. Sample fabrication was carried out in the Microelectronics Research Center at the University of Texas at Austin, which is a member of the National Science Foundation National Nanotechnology Coordinated Infrastructure. We would like to acknowledge stimulating discussions with Li Tao and Craig Prater.

1 Xu XG, Rang M, Craig IM, Raschke MB. Pushing the sample-size limit of infrared vibrational nanospectroscopy: From monolayer toward single molecule sensitivity. J Phys Chem Lett 2012; 3: 1836-1841.

2 Knoll B, Keilmann F. Near-field probing of vibrational absorption for chemical microscopy. Nature 1999; 399: 134-137.

3 Khatib O, Wood JD, McLeod AS, Goldflam MD, Wagner M et al. Graphene-based platform for infrared near-field nanospectroscopy of water and biological materials in an aqueous environment. ACS Nano 2015; 9: 7968-7975.

4 Huth F, Govyadinov A, Amarie S, Nuansing W, Keilmann F et al. Nano-FTIR absorption spectroscopy of molecular fingerprints at $20 \mathrm{~nm}$ spatial resolution. Nano Lett 2012; 12: 3973-3978.

5 Huth F, Schnell M, Wittborn J, Ocelic N, Hillenbrand R. Infrared-spectroscopic nanoimaging with a thermal source. Nat Mater 2011; 10: 352-356.

6 Brehm M, Taubner T, Hillenbrand R, Keilmann F. Infrared spectroscopic mapping of single nanoparticles and viruses at nanoscale resolution. Nano Lett 2006; 6: 1307-1310.

7 Platkov M, Tsun A, Nagli L, Katzir A. A scanning near-field middle-infrared microscope for the study of objects submerged in water. Appl Phys Lett 2008; 92: 104104.

8 Generosi J, Margaritondo G, Sanghera JS, Aggarwal ID, Tolk NH et al. Infrared scanning near-field optical microscopy investigates order and clusters in model membranes. J Microsc 2008; 229: 259-263.

9 Betzig E, Lewis A, Harootunian A, Isaacson M, Kratschmer E. Near field scanning optical microscopy (NSOM): development and biophysical applications. Biophys $J$ 1986; 49: 269-279.

10 Dazzi A, Prazeres R, Glotin F, Ortega JM. Local infrared microspectroscopy with subwavelength spatial resolution with an atomic force microscope tip used as a photothermal sensor. Opt Lett 2005; 30: 2388-2390.

11 Dazzi A, Glotin F, Carminati R. Theory of infrared nanospectroscopy by photothermal induced resonance. J Appl Phys 2010; 107: 124519.

12 Dazzi A, Prater CB, Hu QC, Chase DB, Rabolt JF et al. AFM-IR: combining atomic force microscopy and infrared spectroscopy for nanoscale chemical characterization. Appl Spectrosc 2012; 66: 1365-1384.

13 Policar C, Waern JB, Plamont M-A, Clède S, Mayet C et al. Subcellular IR imaging of a metal-carbonyl moiety using photothermally induced resonance. Angew Chem Int Ed 2011; 50: 860-864.

14 Lahiri B, Holland G, Aksyuk V, Centrone A. Nanoscale imaging of plasmonic hot spots and dark modes with the photothermal-induced resonance technique. Nano Lett 2013; 13: 3218-3224.

15 Felts JR, Cho H, Yu MF, Bergman LA, Vakakis AF et al. Atomic force microscope infrared spectroscopy on $15 \mathrm{~nm}$ scale polymer nanostructures. Rev Sci Instrum 2013; 84: 023709. 
16 Lahiri B, Holland G, Centrone A. Chemical imaging beyond the diffraction limit: experimental validation of the PTIR technique. Small 2013; 9: 439-445.

17 Lu F, Belkin MA. Infrared absorption nano-spectroscopy using sample photoexpansion induced by tunable quantum cascade lasers. Opt Express 2011; 19: 19942-19947.

$18 \mathrm{Lu} \mathrm{F}$, Jin MZ, Belkin MA. Tip-enhanced infrared nanospectroscopy via molecular expansion force detection. Nat Photon 2014; 8: 307-312.

19 Nowak D, Morrison W, Wickramasinghe HK, Jahng J, Potma E et al. Nanoscale chemical imaging by photoinduced force microscopy. Sci Adv 2016; 2: e1501571.

20 Rosu C, Jacobeen S, Park K, Reichmanis E, Yunker P et al. Domed silica microcylinders coated with oleophilic polypeptides and their behavior in lyotropic cholesteric liquid crystals of the same polypeptide. Langmuir 2016; 32: 13137-13148.

21 Amenabar I, Poly S, Goikoetxea M, Nuansing W, Lasch P et al. Hyperspectral infrared nanoimaging of organic samples based on Fourier transform infrared nanospectroscopy. Nat Commun 2017; 8: 14402.

22 Centrone A. Infrared imaging and spectroscopy beyond the diffraction limit. Annu Rev Anal Chem 2015; 8: 101-126.

23 Mayet C, Dazzi A, Prazeres R, Allot F, Glotin F et al. Sub-100 nm IR spectromicroscopy of living cells. Opt Lett 2008; 33: 1611-1613.

24 Algara-Siller G, Lehtinen O, Wang FC, Nair RR, Kaiser U et al. Square ice in graphene nanocapillaries. Nature 2015; 519: 443-445.

25 Hansen WN. Electric fields produced by the propagation of plane coherent electromagnetic radiation in a stratified medium. J Opt Soc Am 1968; 58: 380-390.

26 Inouye Y, Kawata S. Near-field scanning optical microscope with a metallic probe tip. Opt Lett 1994; 19: 159-161.
27 Schäffer TE, Cleveland JP, Ohnesorge F, Walters DA, Hansma PK. Studies of vibrating atomic force microscope cantilevers in liquid. J Appl Phys 1996; 80: 3622-3627.

28 Höppener C, Siebrasse JP, Peters R, Kubitscheck U, Naber A. High-resolution near-field optical imaging of single nuclear pore complexes under physiological conditions. Biophys J 2005; 88: 3681-3688.

29 Max JJ, Chapados C. Isotope effects in liquid water by infrared spectroscopy. III. $\mathrm{H}_{2} \mathrm{O}$ and $\mathrm{D}_{2} \mathrm{O}$ spectra from 6000 to $0 \mathrm{~cm}^{-1}$. J Chem Phys 2009; 131: 184505.

30 Ziegler D, Klaassen A, Bahri D, Chmielewski D, Nievergelt A et al. Encased cantilevers for low-noise force and mass sensing in liquids. Proceedings of 2014 IEEE 27th International Conference on Micro Electro Mechanical Systems (MEMS); 26-30 January 2014; San Francisco, CA, USA. IEEE: San Francisco, CA, USA, 2014; pp 128-131.

(c) (i) (2) This work is licensed under a Creative Commons Attribution BY NG SA NonCommercial-ShareAlike 4.0 International License. The images or other third party material in this article are included in the article's Creative Commons license, unless indicated otherwise in the credit line; if the material is not included under the Creative Commons license, users will need to obtain permission from the license holder to reproduce the material. To view a copy of this license, visit http:// creativecommons.org/licenses/by-nc-sa/4.0/

(C) The Author(s) 2017

Supplementary Information for this article can be found on the Light: Science \& Applications' website (http://www.nature.com/lsa). 